\title{
Electron and Ion Optics
}




\section{MICRODEVICES}

\section{Physics and Fabrication Technologies}

Series Editors: Ivor Brodie and Julius J. Muray

SRI International

Menlo Park, California

\section{ELECTRON AND ION OPTICS \\ Miklos Szilagyi}

\section{GaAs DEVICES AND CIRCUITS}

Michael Shur

SEMICONDUCTOR LITHOGRAPHY

Principles, Practices, and Materials

Wayne M. Moreau 


\section{Electron and Ion Optics}

Miklos Szilagyi

University of Arizona

Tucson, Arizona

Plenum Press • New York and London 
Library of Congress Cataloging in Publication Data

Szilágyi, Miklós.

Electron and ion optics / Miklós Szilágyi.

p. $\mathrm{cm} .-$ (Microdevices)

Bibliography: $p$.

Includes index.

ISBN-13: 978-1-4612-8247-1 e-ISBN-13: 978-1-4613-0923-9

DOI: $10.1007 / 978-1-4613-0923-9$

1. Electron optics. 2. Electron beams. 3. Ion bombardment. 4. Electromagnetic lenses.

I. Title. II. Series.

QC793.5.E62S95 1988

$537.5^{\prime} 6-\mathrm{dc} 19$

$87-32180$

CIP

(C) 1988 Plenum Press, New York

Softcover reprint of the hardcover 1st edition 1988

A Division of Plenum Publishing Corporation

233 Spring Street, New York, N.Y. 10013

All rights reserved

No part of this book may be reproduced, stored in a retrieval system, or transmitted in any form or by any means, electronic, mechanical, photocopying, microfilming, recording, or otherwise, without written permission from the Publisher 
To Jutka, Gabor, and Zoltan

For the time that should have belonged to them 


\section{Preface}

The field of electron and ion optics is based on the analogy between geometrical light optics and the motion of charged particles in electromagnetic fields. The spectacular development of the electron microscope clearly shows the possibilities of image formation by charged particles of wavelength much shorter than that of visible light.

As new applications such as particle accelerators, cathode ray tubes, mass and energy spectrometers, microwave tubes, scanning-type analytical instruments, heavy beam technologies, etc. emerged, the scope of particle beam optics has been extended to the formation of fine probes. The goal is to concentrate as many particles as possible in as small a volume as possible.

Fabrication of microcircuits is a good example of the growing importance of this field. The current trend is towards increased circuit complexity and pattern density. Because of the diffraction limitation of processes using optical photons and the technological difficulties connected with $\mathrm{x}$-ray processes, charged particle beams are becoming popular. With them it is possible to write directly on a wafer under computer control, without using a mask. Focused ion beams offer especially great possibilities in the submicron region. Therefore, electron and ion beam technologies will most probably play a very important role in the next twenty years or so.

Many books have been published on different aspects of the theory and applications of electron and ion optics. This one is intended to be a self-contained, systematic and up-to-date introduction to the field. The reader can find the derivation of the most essential relationships, the understanding of which is absolutely necessary to do any meaningful work with particle beams and optics, but which are usually presented without proof in most books. Modern computer methods are especially emphasized. The book can serve as a textbook for engineers, scientists, and graduate students who wish to understand the basic principles of electron and ion optics and apply them to the design and/or operation of beamtype devices and instruments.

The author is well aware of the fact that it is not easy to reach these goals. One volume is hardly adequate even to cover the theoretical foundations and most important applications. Therefore, the treatment is restricted to the presentation of the basics and the most recent results of research, including the author's own. Throughout the book the relevance of the presented material to practical applications is emphasized, but no attempt has been made to review the applications themselves. We have given over 400 basic references, including 
publications that report on recent developments in the field. Owing to limitations of space, the list of references is very far from being complete.

To study this book no previous acquaintance with physical electronics is required. The necessary introductory information is presented in Chapter 1 . The theoretical material is derived from basic principles. Because of the mathematical nature of the subject, however, a thorough knowledge of calculus (including vector calculus) is presupposed.

The author has been working in this field since 1958. It was a great joy for me to be able to sit down and summarize the basics of my knowledge in the present volume. This knowledge has been accumulated in the course of interaction with numerous people. I am especially greatful to Professors V. M. Kelman, S. Ya. Yavor, and A. D. Sushkov and to the memory of the unforgettable Dennis Gabor. My research work in the last three years has been supported by the National Science Foundation. I appreciate the continuous support of Dean T. Triffet of the University of Arizona, the inspiration of the editors of this series, and the highquality work of Plenum Press. The book would have never been completed without the encouragement, patience, and love of my family.

Tucson, Arizona

Miklos Szilagyi 


\section{Contents}

CHAPTER 1. Introductory Survey

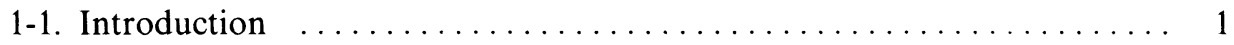

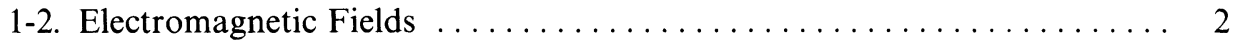

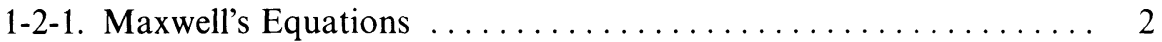

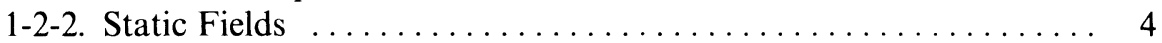

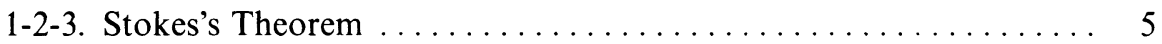

1-3. Some Basic Classical Mechanics $\ldots \ldots \ldots \ldots \ldots \ldots \ldots \ldots \ldots \ldots$

1-3-1. Hamilton's Principle; The Lagrangian Equations of Motion .... 6

1-3-2. The Maupertuis Principle ................... 8

1-4. A Little Reminder of Geometrical Optics $\ldots \ldots \ldots \ldots \ldots \ldots \ldots \ldots \ldots$

1-4-1. Fermat's Principle; The Index of Refraction $\ldots \ldots \ldots \ldots \ldots . \ldots$

1-4-2. Axially Symmetric Lenses ..................... 9

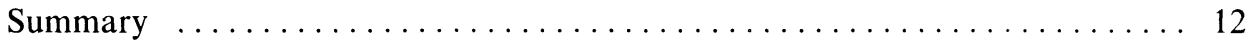

CHAPTER 2. Motion of Charged Particles in Electric and Magnetic Fields

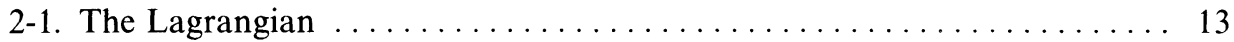

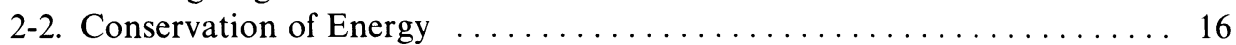

2-2-1. Motion of Free Particles; Velocity versus Potential . . . . . . . . 17

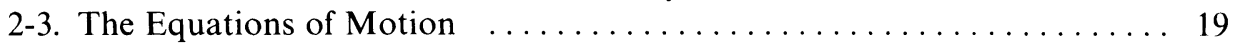

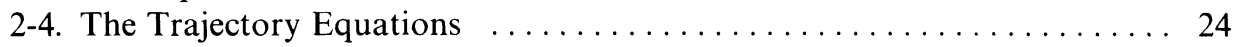

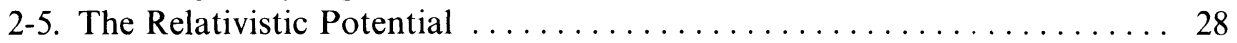

2-6. The Electron Optical Index of Refraction $\ldots \ldots \ldots \ldots \ldots \ldots \ldots \ldots \ldots$

2-7. Particles in Homogeneous Fields ... . . . . . . . . . . . . . . . . 31

2-7-1. The Parallel-Plate Capacitor $\ldots \ldots \ldots \ldots \ldots \ldots \ldots \ldots \ldots \ldots \ldots$

2-7-1-1. Electrostatic Deflection ................ 34

2-7-1-2. A Simple Velocity Analyzer ................ 35

2-7-2. Homogeneous Magnetic Field . . . . . . . . . . . . . . 36

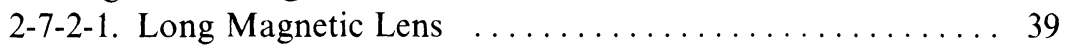

2-7-2-2. Magnetic Deflection ................. 40

2-7-3. The Simultaneous Action of Homogeneous Electric and Magnetic

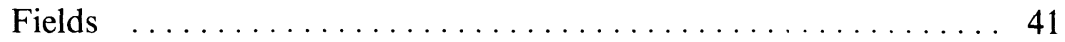

2-7-3-1. Mass Analysis and Other Applications ........... 45 


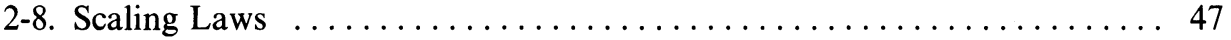

Summary 50

CHAPTER 3. Determination of Electric and Magnetic Fields

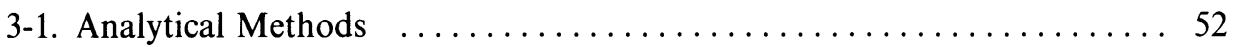

3-1-1. Series Expansions of Potentials and Fields $\ldots \ldots \ldots \ldots \ldots \ldots, 52$

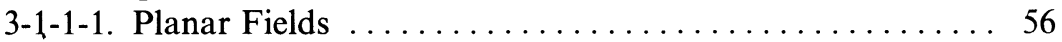

3-1-1-2. Axially Symmetric Fields . . . . . . . . . . . . . 58

3-1-1-3. Multipole Fields ....................... 60

3-1-2. Analytical Calculation of Axially Symmetric Potential Fields .... 67

3-1-2-1. Separation of Variables ................... 67

3-1-2-2. Difficulties of Analytical Calculations (Electrostatic Field of Two Equidiameter Cylinders) $\ldots \ldots \ldots \ldots \ldots \ldots 72$

3-1-2-3. Field of a Circular Aperture ................. 77

3-1-2-4. Rapid Evaluation of Fields Produced by Two or More

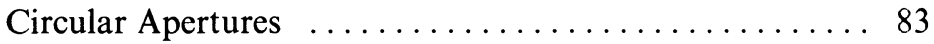

3-1-3. Analytical Calculation of Multipole Fields $\ldots \ldots \ldots \ldots \ldots \ldots .68$

3-1-3-1. Short Multipoles ......................... 86

3-1-3-2. Long Multipoles $\ldots \ldots \ldots \ldots \ldots \ldots \ldots \ldots \ldots \ldots \ldots \ldots$

3-1-3-3. Ideal Multipoles $\ldots \ldots \ldots \ldots \ldots \ldots \ldots \ldots \ldots . \ldots 1$

3-1-3-4. The Method of Conformal Transformation ......... 94

3-1-4. On the Role of Magnetic Materials .................. 96

3-1-5. Analytical Calculation of Magnetic Fields Produced by Currents . 102

3-1-5-1. The Biot-Savart Law . . . . . . . . . . . . . . . . . 102

3-1-5-2. Field of a Straight Wire ..................... 104

3-1-5-3. Field of a Circular Loop . . . . . . . . . . . . . . . . . 104

3-1-5-4. Field of a Thin Solenoid .................... 105

3-1-5-5. Field of a Multilayer Coil . ................. 106

3-1-5-6. Field of a Pancake Coil ...................... 108

3-2. Measurement of Fields and Analog Methods .................. 109

3-2-1. Measurement of Magnetic Fields ..................... 109

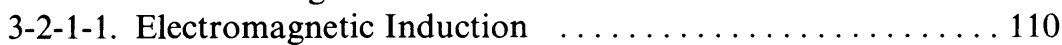

3-2-1-2. Hall Effect ............................... 110

3-2-1-3. Permalloy and Bismuth Probes $\ldots \ldots \ldots \ldots \ldots \ldots \ldots \ldots \ldots 11$

3-2-1-4. Magnetic Resonance .........................111

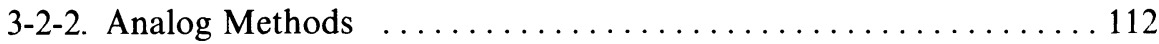

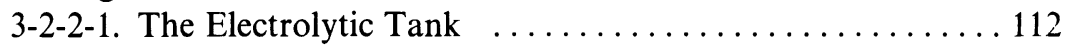

3-2-2-2. The Resistor Network . .................... 114

3-2-2-3. Other Analog Methods . ...................... 119

3-3. Numerical Methods .................................. 119

3-3-1. Accuracy ......................................

3-3-1-1. Errors Due to the Nature of the Problem ........... 120

3-3-1-2. Errors Due to the Number Representation in the Computer .............................. 121

3-3-1-3. Errors Due to the Numerical Method $\ldots \ldots \ldots \ldots \ldots \ldots 122$

3-3-2. The Finite-Difference Method ..................... 122

3-3-2-1. Methods of Solution for Systems of Algebraic Equations . 128 
3-3-3. The Finite-Element Method . . . . . . . . . . . . . . . . . 130

3-3-4. The Charge-Density (Integral) Method . . . . . . . . . . . . 137

3-3-5. Numerical Differentiation and Interpolation . . . . . . . . . 143

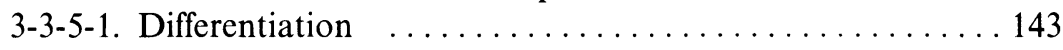

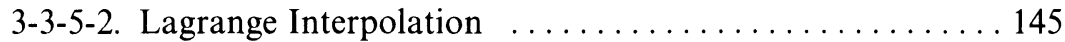

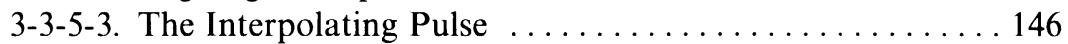

3-3-5-4. The Cubic Spline ... . . . . . . . . . . . . . . 147

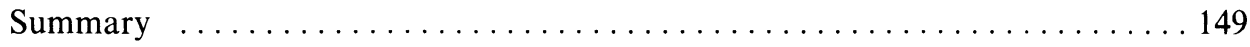

CHAPTER 4. Focusing With Axially Symmetric Fields

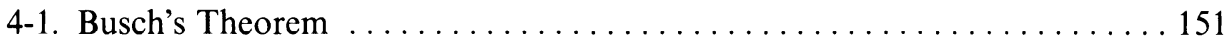

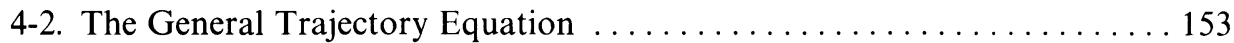

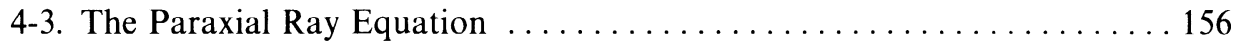

4-4. Image Formation by Paraxial Rays $\ldots \ldots \ldots \ldots \ldots \ldots \ldots \ldots \ldots \ldots \ldots \ldots \ldots \ldots$

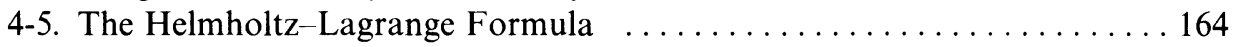

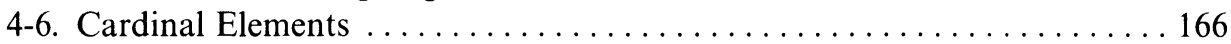

4-6-1. Asymptotic Cardinal Elements . . . . . . . . . . . . . . . 170

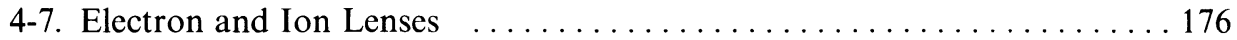

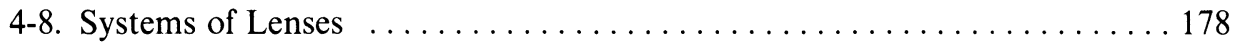

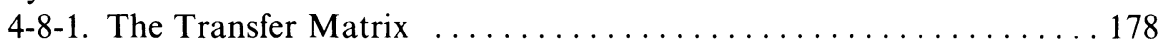

4-8-2. Combination of Two Thick Lenses . . . . . . . . . . . . . 181

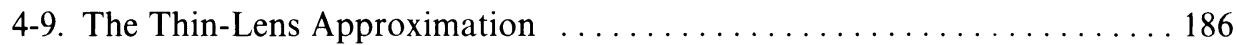

4-9-1. Combination of Thin Lenses . . . . . . . . . . . . . . . . 191

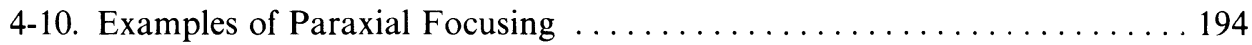

4-10-1. Paraxial Trajectories in Homogeneous Fields . . . . . . . . . . . . 194

4-10-1-1. Homogeneous Electrostatic Field . . . . . . . . . . . . 194

4-10-1-2. Skew Rays ........................ 196

4-10-1-3. Homogeneous Magnetic Field . . . . . . . . . . . . 197

4-10-2. The Single-Loop Magnetic Lens . . . . . . . . . . . . . . . . . 200

4-10-3. Lens Systems ........................... 203

4-10-3-1. Telescopic System ..................... 203

4-10-3-2. Magnification of Lens Systems . . . . . . . . . . . . . 204

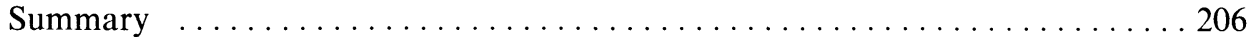

CHAPTER 5. The Theory of Aberrations

5-1. The Method of Characteristic Functions . . . . . . . . . . . . . . . . 208

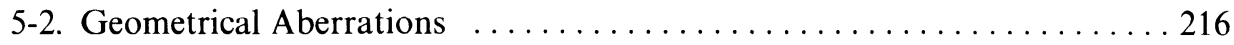

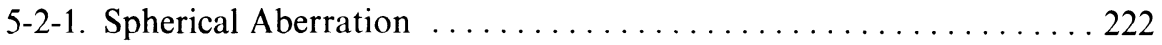

5-2-1-1. Zero and Infinite Magnifications ............. 227

5-2-1-2. Alternative Forms of the Spherical Aberration Coefficient 229

5-2-1-3. Scherzer's Theorem ................... 236

5-2-1-4. The Disk of Minimum Confusion ... . . . . . . . 237

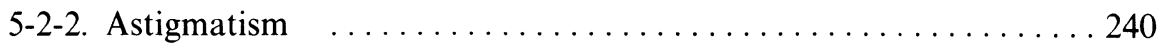

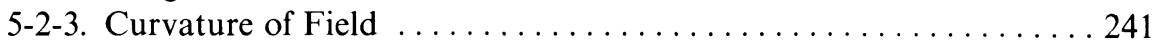

5-2-4. Distortion .............................. 242 
5-2-5. Coma ................................ 244

5-2-6. Anisotropic Aberrations . . . . . . . . . . . . . . . . 245

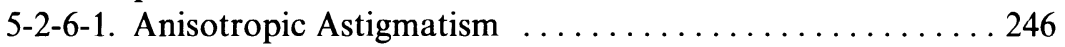

5-2-6-2. Anisotropic Distortion . . . . . . . . . . . . . . 247

5-2-6-3. Anisotropic Coma ..................... 248

5-2-7. On the Relative Importance of the Different Geometrical Aberrations .................................. 250

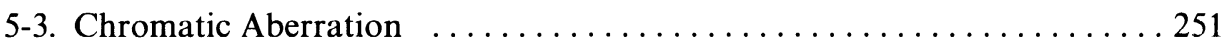

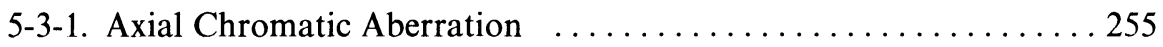

5-3-1-1. Zero and Infinite Magnifications ............ 258

5-3-1-2. The Upper Limit of the Axial Chromatic Aberration . . . 259

5-3-2. Chromatic Aberration of Magnification . . . . . . . . . . 262

5-3-3. Anisotropic Chromatic Aberration ................. 262

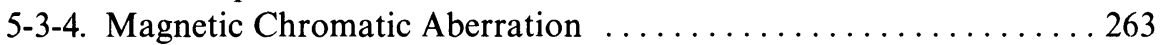

5-4. Asymptotic Aberrations . . . ........................ 264

5-4-1. The Dependence of the Asymptotic Aberration Coefficients on the

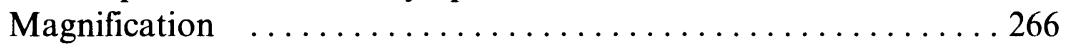

5-4-1-1. Polynomial Expression for the Asymptotic Spherical Aberration Coefficient ................... 267

5-4-1-2. Polynomial Expression for the Asymptotic Axial Chromatic Aberration Coefficient .............271

5-4-2. Aberrations of Thin Lenses . . . . . . . . . . . . . . . . . . 274

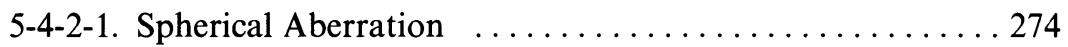

5-4-2-2. Axial Chromatic Aberration ................ 275

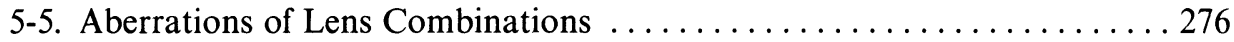

5-5-1. Addition of Spherical Aberrations . . . . . . . . . . . . . . . 277

5-5-2. Addition of Axial Chromatic Aberrations . . . . . . . . . . . . 280

5-6. Other Sources of Aberrations and Aberration Correction ........... 281

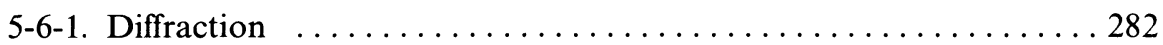

5-6-2. Space Charge and Surface Charges ................ 283

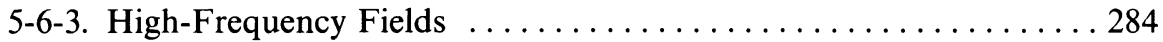

5-6-4. Lack of Axial Symmetry ... . . . . . . . . . . . . . . . . . 284

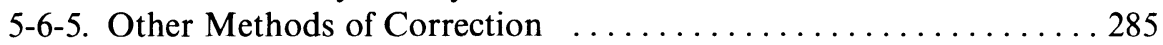

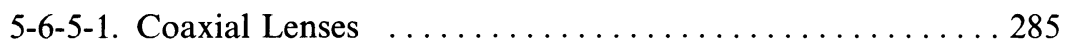

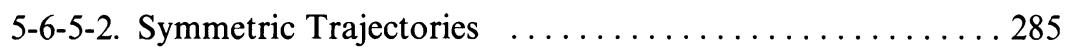

5-6-5-3. Position of the Limiting Aperture $\ldots \ldots \ldots \ldots \ldots \ldots 285$

5-6-5-4. Digital Image Processing ................ 286

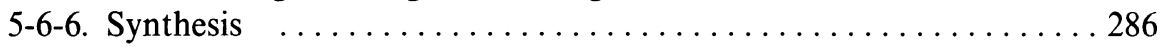

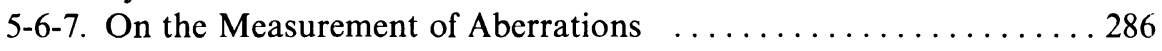

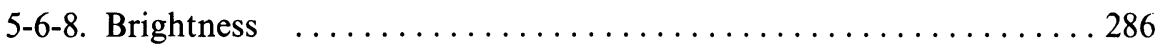

5-7. Simultaneous Action of Different Aberrations . . . . . . . . . . . . . 289

5-7-1. Negligibly Small Sources . . . . . . . . . . . . . . . . . . . 290

5-7-2. Finite Sources . . . . . . . . . . . . . . . . . . . 291

5-7-2-1. Negligible Chromatic Aberration .............. 292

5-7-2-2. Negligible Spherical Aberration ................ 294

5-7-3. Aberration Mixing for Lens Combinations . . . . . . . . . . . 295

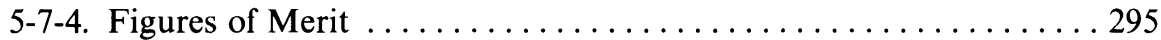

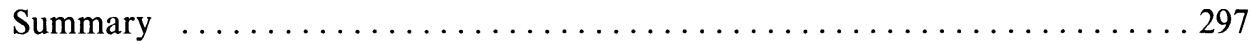


CHAPTER 6. Numerical Techniques for Ray Tracing and Calculation of Aberrations

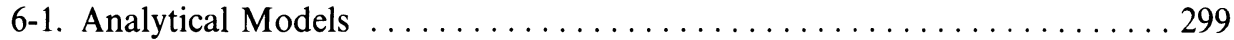

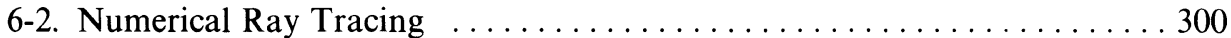

6-2-1. The Runge-Kutta Method ... . . . . . . . . . . . . . . . . . . . . 302

6-2-2. Multistep Methods .......................... 306

6-2-2-1. Numerov's Method .................... 307

6-2-3. Additional Remarks on Accuracy . . . . . . . . . . . . . . . . . 307

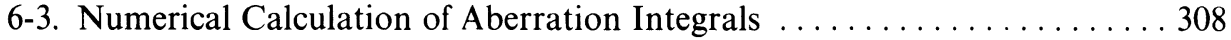

6-3-1. Trapezoidal Integration . . . . . . . . . . . . . . . . . . . . . . . . 309

6-3-2. Simpson's Rule . . . . . . . . . . . . . . . . . . . . . . . . . 310

6-3-3. Romberg Integration and the Gaussian Quadrature . . . . . . . 311

Summary

\section{CHAPTER 7. Electrostatic Lenses}

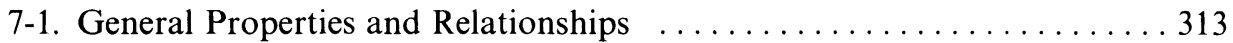

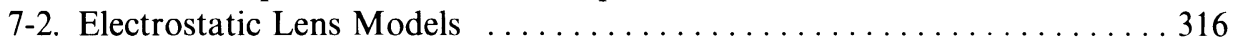

7-2-1. Analytical Models . . . . . . . . . . . . . . . . . . 316

7-2-2. The Piecewise Linear Model .................... 316

7-2-3. The Piecewise Quadratic Model . . . . . . . . . . . . . . . . . 317

7-2-4. The Spline Model ......................... 320

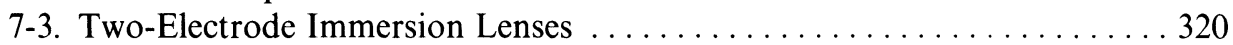

7-3-1. Geometrically Symmetric Lenses . . . . . . . . . . . . . . . . 322

7-3-1-1. A Linear Model ... . . . . . . . . . . . . . . . . . . . 322

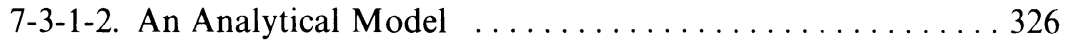

7-3-1-3. The Two-Cylinder Lens .................... 331

7-3-1-4. The Double-Aperture Lens . . . . . . . . . . . . 343

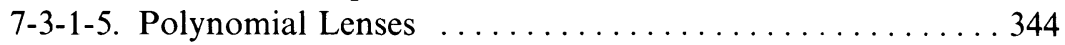

7-3-2. Asymmetric Lenses . . . . . . . . . . . . . . . . . . . . . . 348

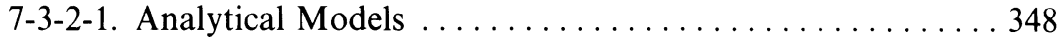

7-3-2-2. The Asymmetric Two-Cylinder Lens . . . . . . . . . . . 350

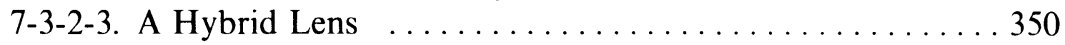

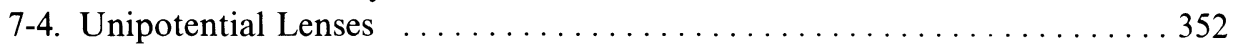

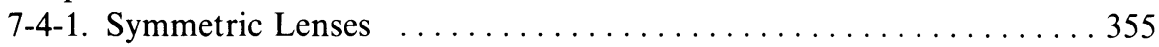

7-4-1-1. A Piecewise Linear Model . . . . . . . . . . . . . . . . . 355

7-4-1-2. A Piecewise Quadratic Model . . . . . . . . . . . . . . . 359

7-4-1-3. An Analytical Model . . . . . . . . . . . . . . . 360

7-4-1-4. The Three-Cylinder Lens ... . . . . . . . . . . . . . 364

7-4-1-5. The Triple-Aperture Lens . . . . . . . . . . . . . 369

7-4-1-6. Other Types of Symmetric Lenses . . . . . . . . . . . 369

7-4-2. Asymmetric Lenses . . . . . . . . . . . . . . . . . . . . . . . 370

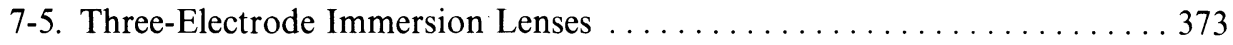

7-5-1. Geometrically Symmetric Lenses . . . . . . . . . . . . . . . . 373

7-5-1-1. The Three-Cylinder Lens . . . . . . . . . . . . . 374

7-5-1-2. Other Types of Geometrically Symmetric Lenses . . . . . . 379

7-5-2. Asymmetric Lenses . . . . . . . . . . . . . . . . . . . . . . . . . . 379 
7-6. Multielectrode Lenses . . . . . . . . . . . . . . . . . . . . . . . . . . 380

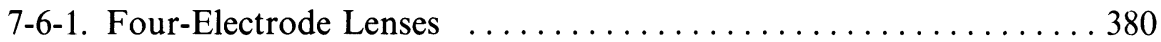

7-6-2. Lenses with Five or More Electrodes . . . . . . . . . . . . 382

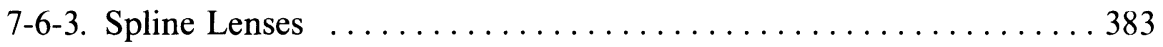

7-7. Comparison of Different Electrostatic Lenses . . . . . . . . . . . . 384

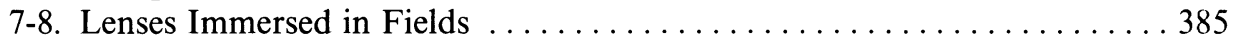

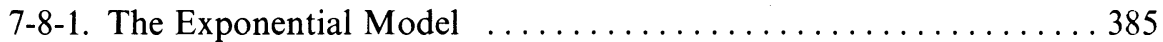

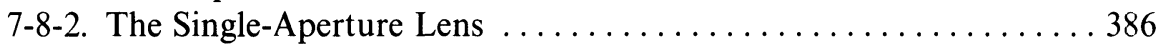

7-8-3. Cathode Lenses, Electron and Ion Sources ............ 388

7-8-3-1. Thermionic Guns . . . . . . . . . . . . . . . . . 390

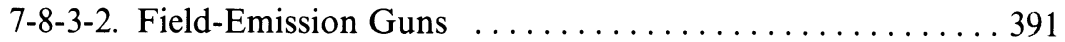

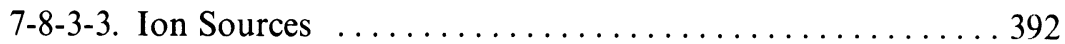

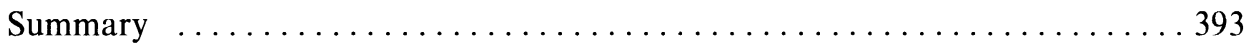

CHAPTER 8. Magnetic Lenses

8-1. General Properties and Relationships $\ldots \ldots \ldots \ldots \ldots . \ldots \ldots 5$

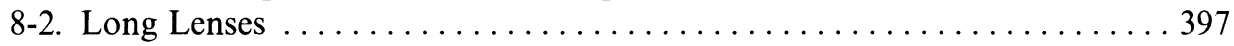

8-2-1. Homogeneous Magnetic Fields . . . . . . . . . . . . . . . . 397

8-2-2. Linear Magnetic Fields . . . . . . . . . . . . . . . . . . . . . . . 398

8-2-3. Long Lenses with Low Spherical Aberration .............. 401

8-3. Magnetic Lens Models . . . . . . . . . . . . . . . . . . . . 401

8-3-1. The Rectangular Model .................... 401

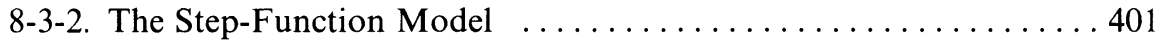

8-3-3. The Piecewise Linear Model . . . . . . . . . . . . . . . . . . . . . . . . 402

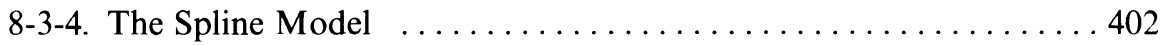

8-3-5. Glaser's Bell-Shaped Model . . . . . . . . . . . . . . . . . . . 402

8-3-5-1. Generalization of the Bell-Shaped Model . . . . . . . 410

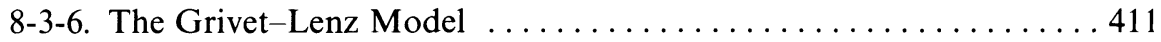

8-3-7. Other Models ......................... 412

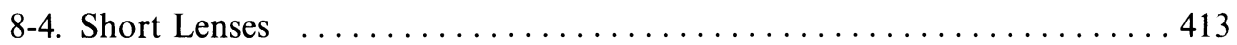

8-4-1. Conventional Lenses . . . . . . . . . . . . . . . . . . . 413

8-4-2. Unconventional Lenses . . . . . . . . . . . . . . . . . . . . 419

8-4-2-1. Superconducting Lenses ............... 419

8-4-2-2. Reduction of the Coil Size by Other Means . . . . . . . . 419

8-4-2-3. Rotation-Free Miniature Lenses . . . . . . . . . . . . . . 419

8-4-2-4. Iron-Free Magnetic Lenses . . . . . . . . . . . . 420

8-4-2-5. Single Pole-Piece Lenses . . . . . . . . . . . . . 420

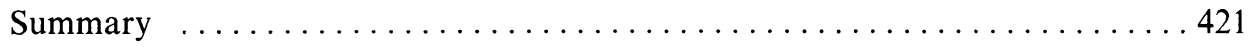

CHAPTER 9. Computer-Aided Optimization and Synthesis of Electron and Ion Lenses

9-1. Is Aberrationless Electron/Ion Optics Possible? ............... 423

9-1-1. The Lower Limit of the Axial Chromatic Aberration of Magnetic

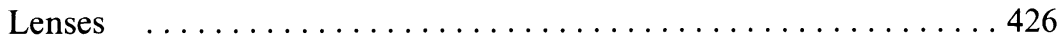

9-2. Optimization: Synthesis versus Analysis $\ldots \ldots \ldots \ldots \ldots \ldots \ldots \ldots \ldots \ldots$ 


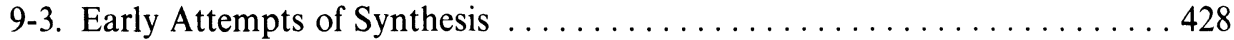

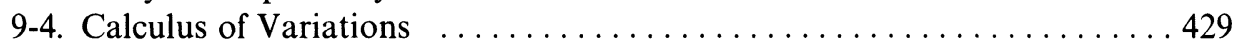

9-4-1. The Lower Limits of the Spherical and Axial Chromatic Aberration Coefficients .......................... 432

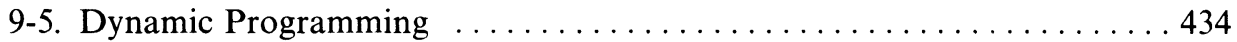

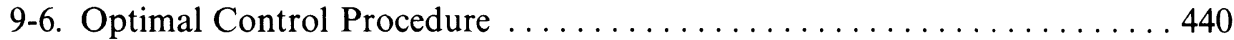

9-7. Analytical Functions .............................. 441

9-8. Reconstruction of Electrodes and Pole Pieces from the Optimized Axial

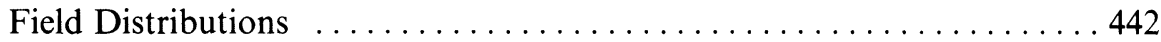

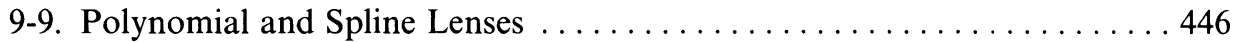

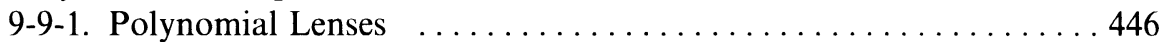

9-9-2. Spline Lenses . ........................... 447

9-9-2-1. Two-Interval Spline Lenses . . . . . . . . . . . . . . . . 449

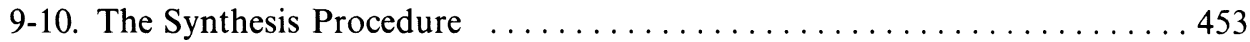

9-10-1. Application: Unconventional Electrostatic Lenses . . . . . . . 456

9-11. Artificial Intelligence Techniques $\ldots \ldots \ldots \ldots \ldots \ldots \ldots \ldots \ldots . \ldots . \ldots . \ldots . \ldots 49$

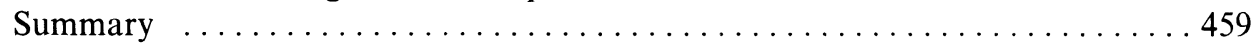

CHAPTER 10. Multipole Lenses

10-1. The Fields of Multipole Lenses $\ldots \ldots \ldots \ldots \ldots \ldots \ldots \ldots \ldots \ldots \ldots \ldots$. . . . 461

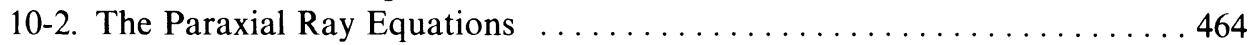

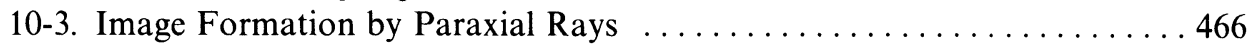

10-4. Systems of Quadrupoles ............................ 468

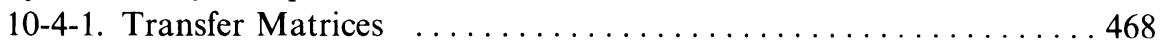

10-4-2. Thin-Lens Representation $\ldots \ldots \ldots \ldots \ldots \ldots \ldots \ldots . \ldots . \ldots . \ldots 49$

10-4-3. Doublets ................................ 471

10-4-4. Triplets ................................ 474

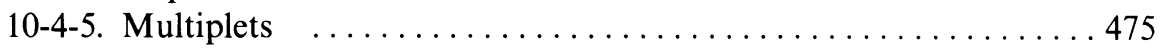

10-4-5-1. Beam Matching ........................ 475

10-5. Aberrations of Multipole Lenses ........................ 476

10-5-1. Geometrical Aberrations $\ldots \ldots \ldots \ldots \ldots \ldots \ldots \ldots . \ldots . \ldots . \ldots 46$

10-5-2. Correction of Aberrations by Means of Multipoles . . . . . . . . 477

10-5-3. Chromatic Aberration .......................... 478

10-5-3-1. The Achromatic Quadrupole Lens ............. 478

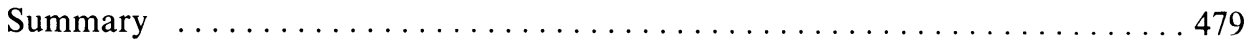

CHAPTER 11. Beam Deflection

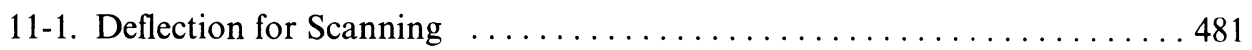

11-1-1. Electrostatic Deflection Fields .................... 481

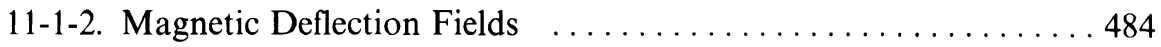

11-1-3. Stigmatic Imaging with Small Deflection ............... 485

11-1-4. Deflection Aberrations .......................... 487

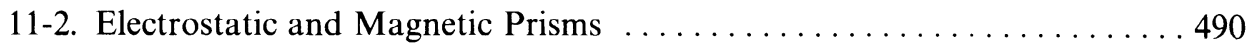

11-2-1. Electrostatic Prisms . . . . . . . . . . . . . . . . . . . . . . 490

11-2-2. Magnetic Prisms . ............................. 493 
11-3. New Symmetries- New Possibilities ...................... 494

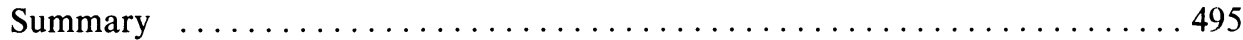

CHAPTER 12. High-Intensity Beams

12-1. Space-Charge Optics ............................ 497

12-1-1. Space-Charge Forces . . . . . . . . . . . . . . . . . . . . . . 499

12-1-1-1. The Electrostatic Force ... . . . . . . . . . . . 499

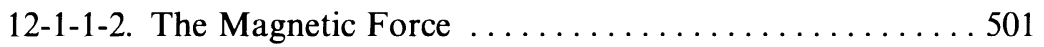

12-1-2. Beam Spreading . . . . . . . . . . . . . . . . . . . 502

12-1-3. Production of High-Intensity Beams . . . . . . . . . . . 505

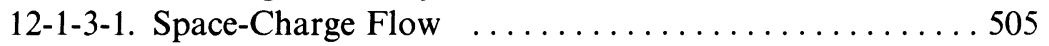

12-1-3-2. The Pierce Gun . . . . . . . . . . . . . . 506

12-1-4. Maintenance of High-Intensity Beams . . . . . . . . . . . . . . 508

12-1-4-1. Focusing by Homogeneous Magnetic Fields . . . . . . 508

12-1-4-2. Periodic Focusing . . . . . . . . . . . . . . . . 510

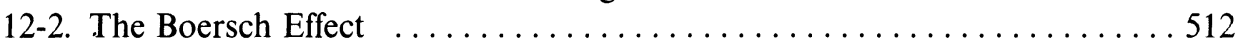

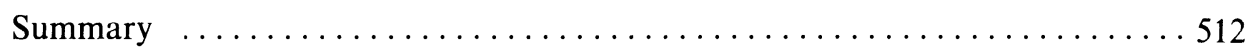

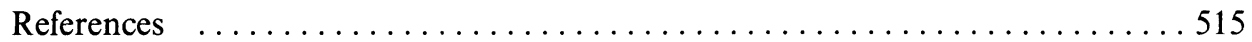

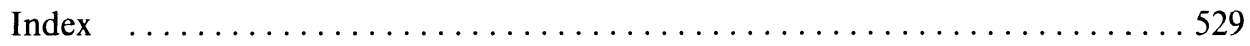

